\title{
Die Notwendigkeit der Einrichtung eines OP-Koordinators
}

\author{
Ergebnisse einer Befragung an 26 Kliniken Bayerns mit mindestens sieben Fachabteilungen \\ und Krankenhäusern der Versorgungsstufe III
}

Hans Jürgen Stopora

\section{Zusammenfassung}

Die Vorteile, die sich durch Einrichtung eines OP-Koordinators für größere OP-Einheiten ergeben, liegen deutlich auf der Hand: Es soll ein bestmöglicher Einsatz der personellen Ressourcen sowie der OP-Raumkapazitäten erreicht werden. Allerdings machen die Ergebnisse der Untersuchung auch deutlich, dass einige Voraussetzungen gegeben sein müssen. Zuständig sollte eine Berufsgruppe im OP sein, die über den Gesamtüberblick verfügt. Durch eine Dienstanweisung sowie klare Unterstellungsverhältnisse wird dem OP-Koordinator eine gewisse Rückendeckung in Konfliktfällen zugeteilt. Der OP-Koordinator sollte für seine Aufgabe freigestellt sein und nicht nur über eine natürliche Autorität und die notwendige Berufserfahrung, sondern auch über menschliche Qualitäten und Kommunikationsfähigkeit verfügen, um im OP-Team anerkannt zu werden. Die Einbeziehung von personellen Gege-

\section{Zuständigkeit für die Koordination}

Überwiegend die Berufsgruppen Anästhesie bzw. Chirurgie regeln die Koordination des OP-Programmes, aber auch die OP-Pflege (Leitung) ist zu $68 \%$ daran beteiligt. Nach Meinung der Befragten sollte an erster Stelle die Anästhesie, gefolgt von der OP-Pflegeleitung die Reihenfolge und Einteilung der OP-Programmpunkte für die einzelnen OP-Säle festlegen.
OP-JOURNAL 2002; 17: 60-63

(C) Georg Thieme Verlag Stuttgart - New York benheiten und Flexibilität in der Saalnutzung trägt zu einem reibungslosen Ablauf wesentlich bei. Und nicht zuletzt: Für die Patienten besteht ein geringeres Risiko, unverhofft vom OPTagesprogramm abgesetzt zu werden und somit eine Verbesserung der Ergebnisqualität.

Die Ergebnisse einer Umfrage unter 26 bayerischen Krankenhäusern der Versorgungsstufe III zeigen, dass sich die Befragten überwiegend für den Einsatz eines verantwortlichen Koordinaaussprechen. Befragt wurden Pflegedirektorinnen. Von 26 ausgegebenen Fragebögen erhielten wir 16 Bögen zurück; das entspricht einer Rücklaufquote von $61,5 \%$.

Obwohl 68\% der Kliniken angeben, bereits über einen OP-Koordinator zu verfügen, sehen über die Hälfte der Befragten ihre OP-Koordination als verbesserungsfähig an. tionsarztes für das OP-Management

Eine von der Klinikleitung erlassene Dienstanweisung für den OP-Koordinator, welche Kompetenzen, Aufgaben und Befugnisse festlegt, ist bei $50 \%$ der Befragten vorhanden; 93\% aller Befragten geben an, dass die Weisungsbefugnis in Form einer Dienstanweisung vorliegen sollte.

Aufgaben und Weisungsbefugnis des OP-Koordinators sollten in einer Dienstanweisung geregelt sein.

\section{Personalressourcen}

Wie notwendig sehen die Befragten die Einbeziehung von Personalressourcen und die Einhaltung einer Anmeldefrist? Mit der OP- bzw. Anästhesieleitung wurde das OP-Programm des nächsten Tages bzw. die Saalnutzung in $43 \%$ regelmäßig besprochen; in $37 \%$ der Fälle überwiegend und gelegentlich in $18 \%$.

Die Bekanntgabe der Vorplanung erfolgte meist zwischen 14 und $15 \mathrm{Uhr}$; die endgültige Planung erfolgte zwischen 14 und 16 Uhr mit Schwerpunkt auf 15 Uhr. Eine Berücksichtigung von äußeren Einflussfaktoren wie Krankenstand des Personals sowie Kapazität der Intensivbetten fand nur in 19\% regelmäßig und überwiegend statt; bei $38 \%$ der Befragten fand eine gelegentliche Berücksichtigung besonderer Situationen statt.

\section{Dienstanweisung}

Die Frage nach der dienstlichen Unterstellung des OP-Koordinators wurde unterschiedlich beantwortet. Häufig war der ärztliche Direktor der Dienstvorgesetzte; aber auch die Pflegedienstleitung sowie die Krankenhausleitung wurden genannt. Die Befragten wünschten sich am häufigsten eine direkte Unterstellung des OP-Koordinators durch die Krankenhausleitung - wahrscheinlich, um im Konfliktfall die notwendige Rückendeckung zu gewährleisten.
Eine direkte Unterstellung des OP-Koordinators durch die Krankenhausleitung gewährleistet die notendige Rückendeckung.

\section{Nutzung der OP-Säle}

Fast die Hälfte der Befragten gab an, ihre OP-Säle flexibel zu nutzen; aber auch die ausschließlich fachgebundene Nutzung der OP-Säle wurde angegeben. Die mehrheitliche Meinung jedoch (81\%) war, dass eine flexible Nutzung der OP-Säle wünschenswert wäre. 


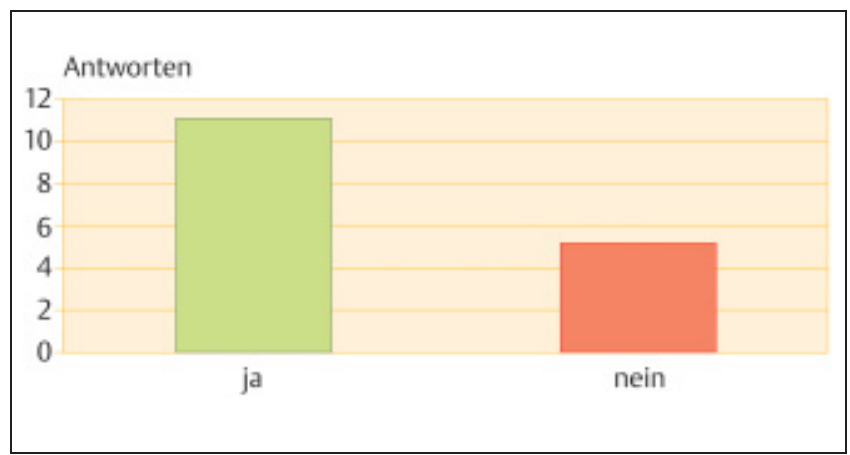

Abb.1 Gibt es in Ihrem Haus die Funktion „OP-Koordinator“?

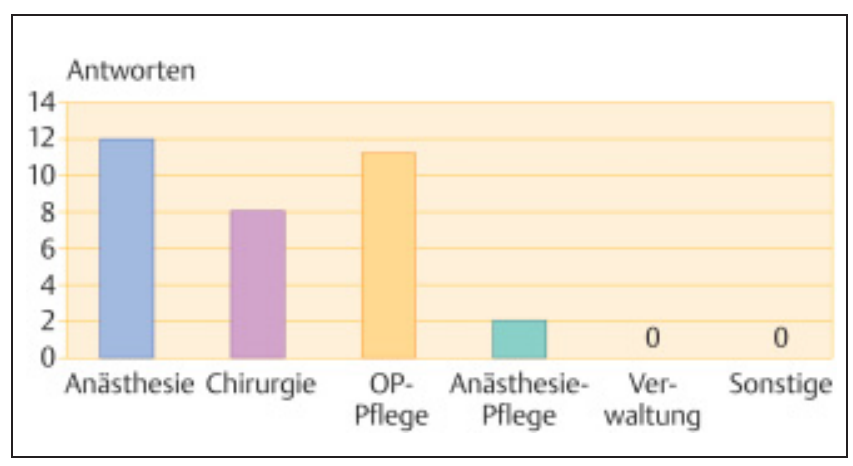

Abb. 3 Wer regelt in Ihrem Haus die Koordination von OP-Programm und OP-Sälen?

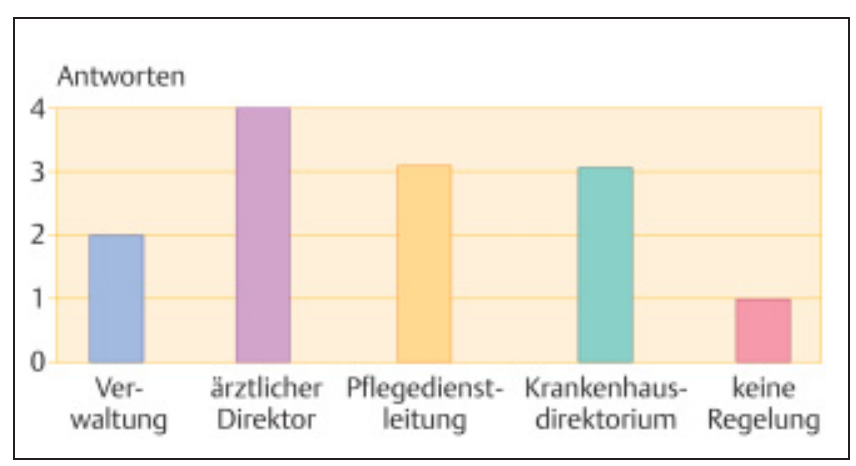

Abb.5 Wem ist der OP-Koordinator direkt unterstellt?

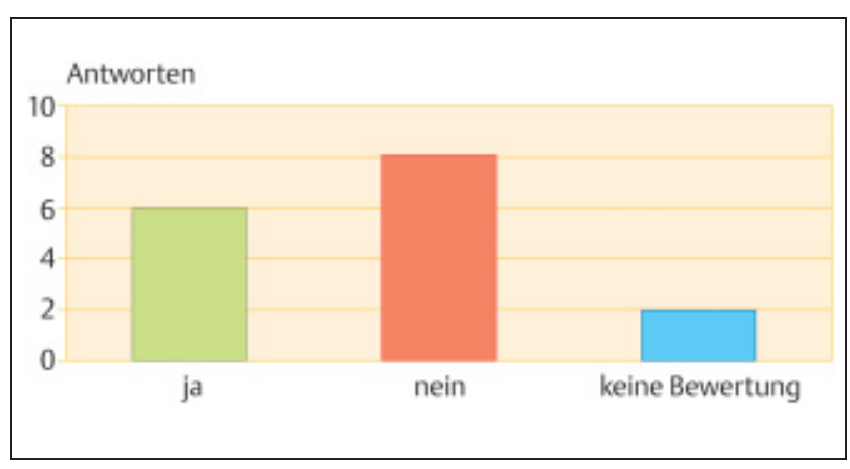

Abb. 7 Ist die Weisungsbefugnis des OP-Koordinators in einer Dienstanweisung fixiert?

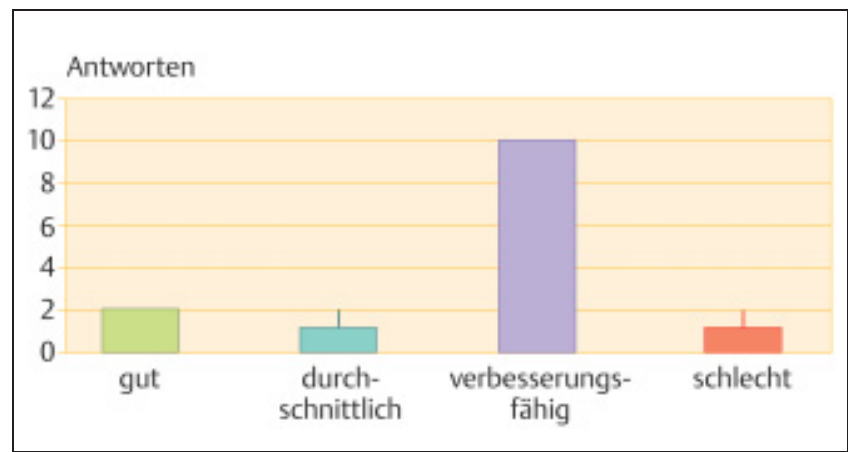

Abb. 2 Wie schätzen Sie die OP-Koordination in Ihrem Haus ein?

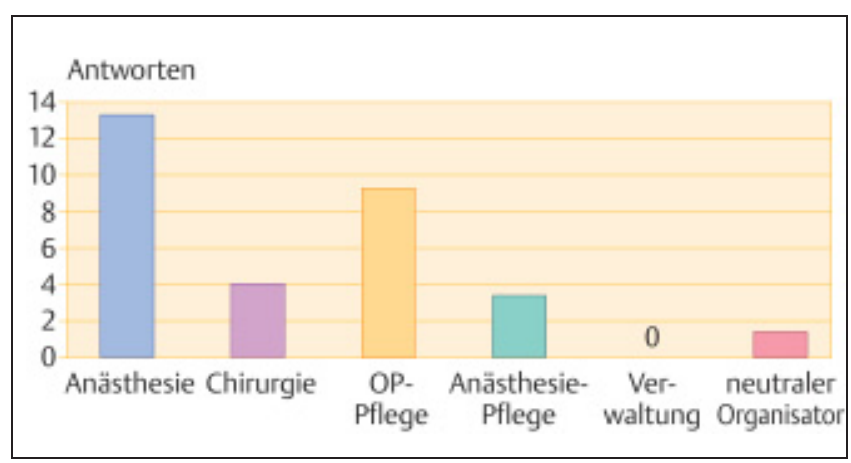

Abb.4 Wer sollte Ihrer Meinung nach die Koordination von OP-Programm/Sälen durchführen?

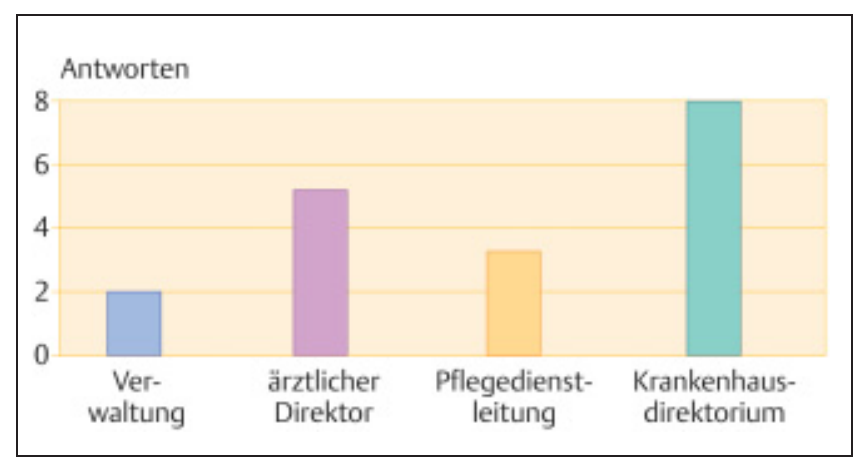

Abb. 6 Wann sollte Ihrer Meinung nach der OP-Koordinator direkt unterstellt werden?

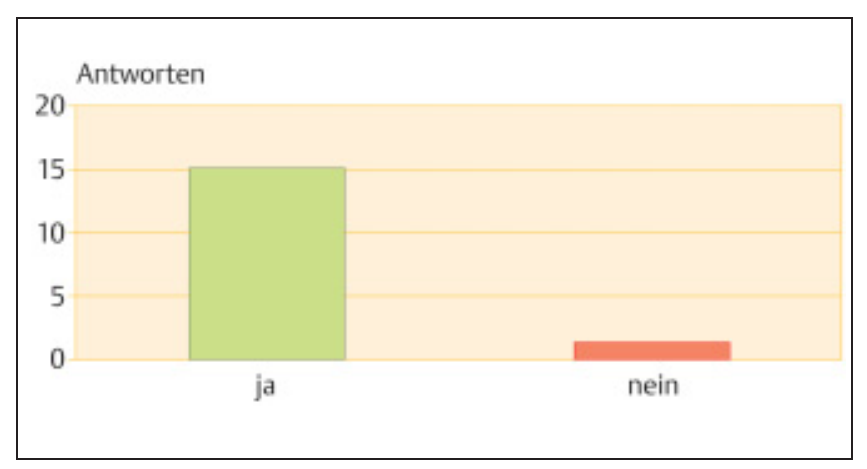

Abb. 8 Sollte die Weisungsbefugnis des OP-Koordinators in einer Dienstanweisung fixiert sein? 


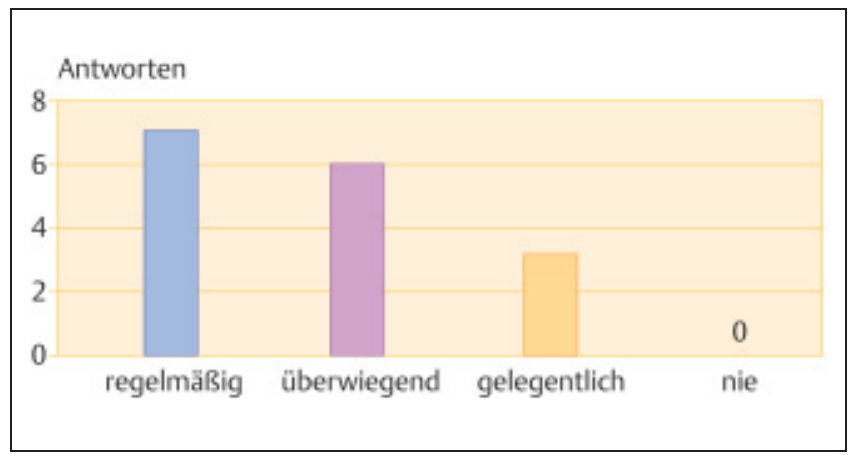

Abb.9 Wird das Programm bzw. die Saalnutzung mit dem Itd. Pflegepersonal OP/AN besprochen?

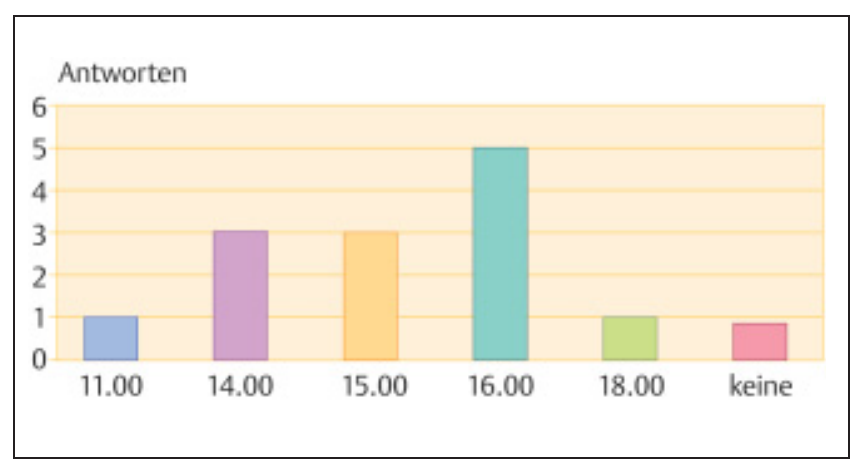

Abb.11 Wann wird die endgültige Planung des OP-Programmes spätestens bekanntgegeben?

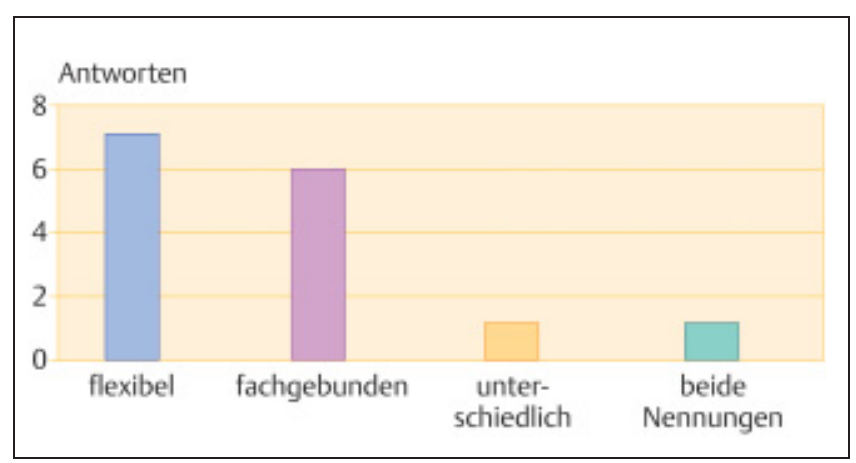

Abb.13 Wie erfolgt die Nutzung Ihrer OP-Säle?

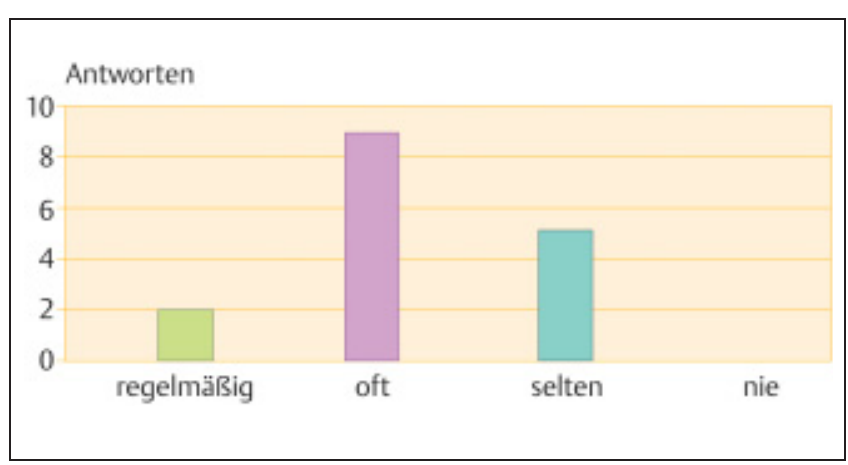

Abb.15 Werden in Ihrem Haus Elektiveingriffe außerhalb der Regelarbeitszeit durchgeführt?

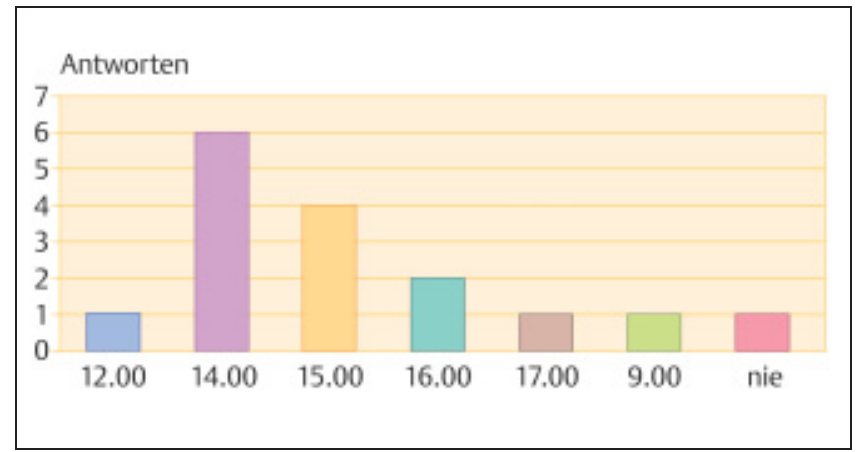

Abb.10 Wann wird die Vorplanung des OP-Programmes spätestens bekanntgegeben?

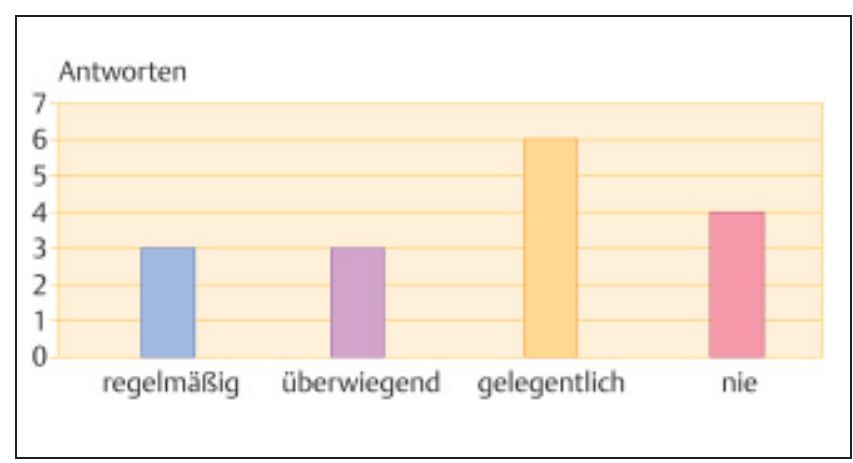

Abb.12 Werden besondere Situationen (z. B. erhöhter Krankenstand belegte Intensivbetten etc. ...) bei der OP-Planung berücksichtigt?

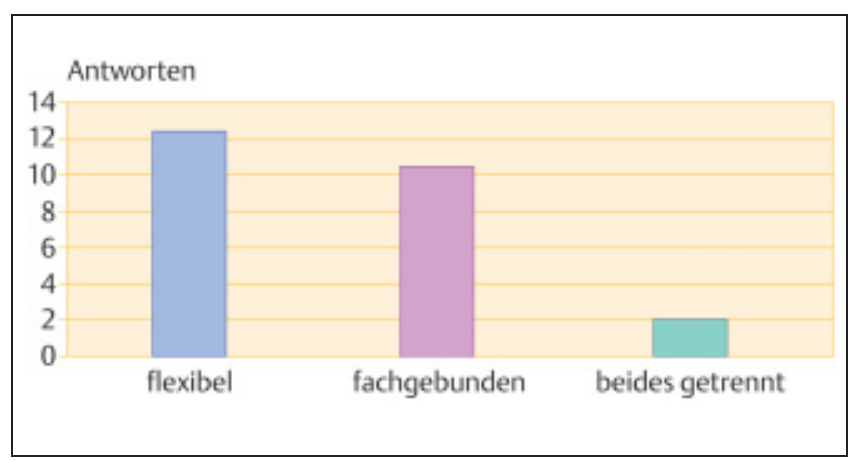

Abb.14 Wie sollte Ihrer Meinung nach die Nutzung der OP-Säle erfolgen?

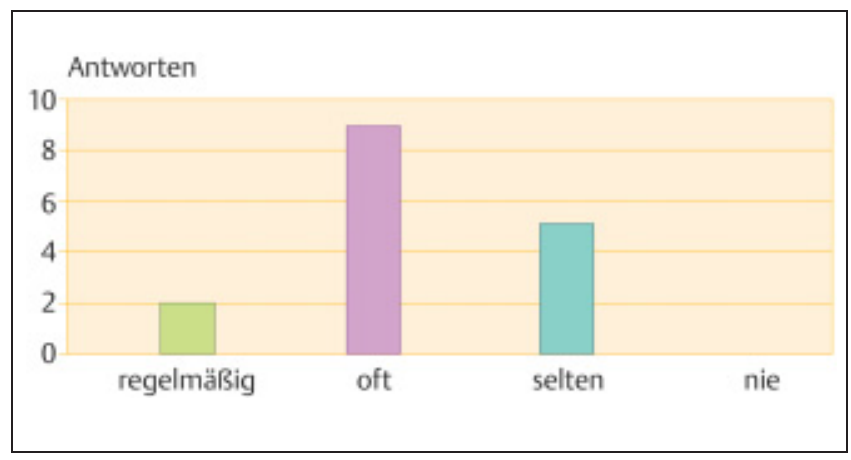

Abb.16 Wären Elektiveingriffe außerhalb der Regelarbeitszeiten durch adäquate Koordination vermeidbar? 


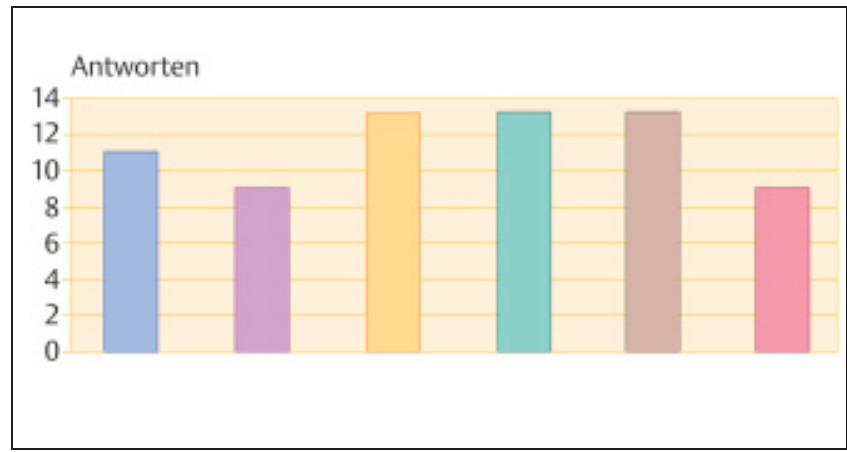

Abb.17 Welche Folgen hätte Ihrer Meinung nach eine Verbesserung der OP-Koordination (Mehrfachnennung möglich)?

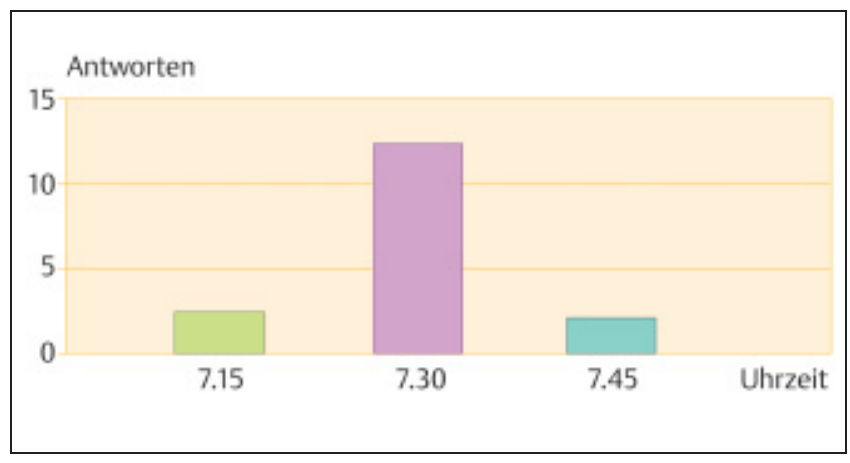

Abb.18 Wann erfolgt in Ihrem Haus der Arbeitsbeginn von OP- und AN-Pflegepersonal?

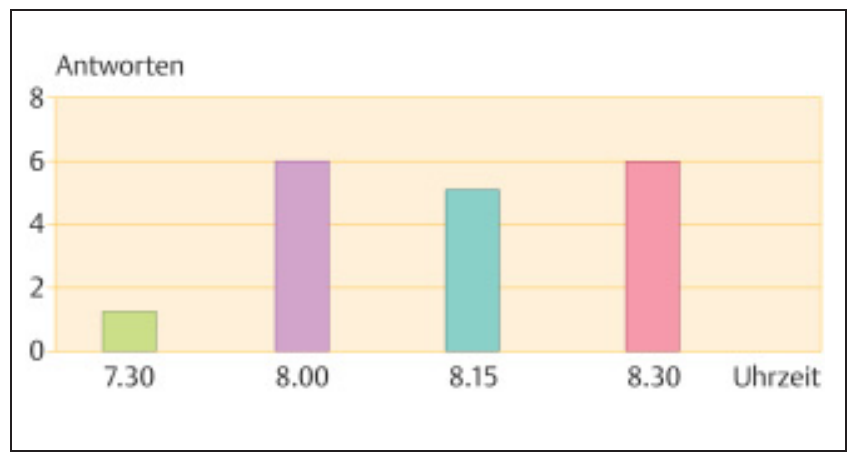

Abb.19 Wann erfolgt in der Regel der Hautschnitt des ersten Programmpunktes/Saal?

\section{Tagesprogramm und Bereitschaftsdienst}

Die Tendenz, Elektiveingriffe außerhalb der Regelarbeitszeit durchzuführen, ist eindeutig zu erkennen. So gaben 56\% der Befragten an, dass dies oft vorkomme. $31 \%$ gaben eine seltene Überschreitung der Regelarbeitszeit an, $13 \%$ die regelmäßige Überschreitung.

Auf die Frage, ob durch adäquate Koordination und Einteilung eine Überschreitung der Regelarbeitszeiten zu vermeiden gewesen wäre, gaben $56 \%$ der Befragten an, dass dies oft zu vermeiden wäre.

Ein erfolgreicher OP-Koordinator besitzt langjährige Berufserfahrung, einen umfassenden Überblick und seine Persönlichkeit wird von allen Operateuren akzeptiert.

\section{Arbeitszeitbeginn und Hautschnitt}

Der Arbeitszeitbeginn von OP- und Anästhesiepflegepersonal konzentriert sich bei $75 \%$ der Befragten auf die Zeit um 7.30 Uhr; dieser Zeitpunkt wird bei den anderen Befragten nur unwesentlich unter bzw. überschritten. Schnittbeginn ist in der Regel zwischen 8 Uhr und 8.30 Uhr.

\section{Effekte einer verbesserten OP-Koordination}

Die Befragten sehen in einer Verbesserung der OP-Koordination folgende positive Effekte:

- Optimierung des Personaleinsatzes durch Wegfall von Mehrarbeits-/Überstunden

- Optimierung der Regelarbeitszeit durch Vermeidung von Wartezeiten

- Steigerung der Motivation im Arzt/ Pflegebereich

- Verbesserung der ärztlichen Stationsversorgung

- Geringere Kosten durch Wegfall von Überstundenzuschlägen

- Verbesserung des Image der Klinik

\section{Hans-Jürgen Stopora}

Pflegedienstleiter

Klinikum Memmingen

Bismarckstraße 23

D-87700 Memmingen 